\title{
Inhaltsübersicht zu Band 1
}

Wichtiger Hinweis — $\mathrm{V}$

Vorwort - VII

I Welle und Teilchen -1

Übersicht über die Ergänzungen zu Kapitel I — 35

$A_{\text {I }} \quad$ De-Broglie-Wellenlängen - 36

B I Zur Unschärferelation - 39

$C_{\text {I }} \quad$ Unschärferelationen und Atomparameter - 41

DI Ein Experiment zur Unschärferelation - 44

$\mathrm{E}_{\mathrm{I}} \quad$ Ein zweidimensionales Wellenpaket -47

$F_{1} \quad$ Zusammenhang zwischen ein- und dreidimensionalen

Problemen -51

G Eindimensionales Gaußsches Wellenpaket - 56

$\mathrm{H}_{\mathrm{I}} \quad$ Stationäre Zustände eines Teilchens in einem eindimensionalen Rechteckpotential - 62

J Wellenpaket an einer Potentialstufe -74

$\mathrm{K}_{\mathrm{I}} \quad$ Aufgaben -81

II Der mathematische Rahmen — 85

Übersicht über die Ergänzungen zu Kapitel II — 158

A II Schwarzsche Ungleichung - 159

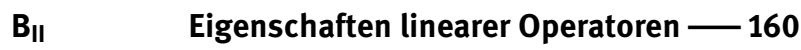

$C_{\text {II }} \quad$ Unitäre Operatoren 170

DII $\quad$ Orts- und Impulsdarstellung - 177

$E_{\text {II }} \quad$ Eigenschaften zweier Observabler mit dem Kommutator i $\hbar-181$



G I $\quad$ Zweidimensionaler unendlich tiefer Potentialtopf 193

$\mathrm{H}_{\mathrm{II}} \quad$ Aufgaben -197 
III Die Postulate der Quantenmechanik — 205

Übersicht über die Ergänzungen zu Kapitel III — 262

$A_{\text {III }} \quad$ Teilchen in einem unendlich tiefen Potentialtopf -264

B III Wahrscheinlichkeitsstrom. Spezialfälle -274

$C_{\text {III } \quad \text { Standardabweichung konjugierter Observabler }-280}$

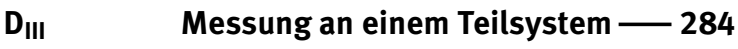

$\mathrm{E}_{\text {III }} \quad$ Der Dichteoperator -289

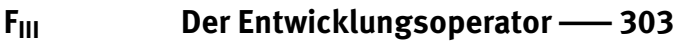

$\mathbf{G}_{\text {III }} \quad$ Schrödinger- und Heisenberg-Bild - 307

$\mathrm{H}_{\text {III }} \quad$ Eichinvarianz -310

JIII Der Propagator der Schrödinger-Gleichung - 325

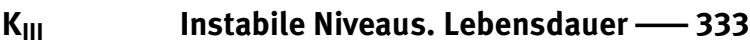

LIIII $\quad$ Aufgaben -337

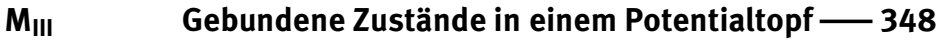

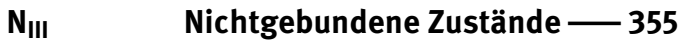

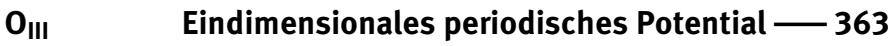

IV Einfache Systeme - 381

Übersicht über die Ergänzungen zu Kapitel IV — 410

A IV Die Pauli-Matrizen - 411

BIV Diagonalisierung einer hermiteschen $2 \times 2$-Matrix -415

$\mathrm{C}_{\text {IV }} \quad$ System mit zwei Niveaus. Fiktiver Spin -419

DIV Systeme mit zwei Spins 1/2 - 425

EIV Dichtematrix für einen Spin 1/2 432

FIV Magnetische Resonanz -437

G Modell des Ammoniakmoleküls - 450

$\mathrm{H}_{\text {IV }} \quad$ Kopplung zwischen stabilem und instabilem Zustand -465

JIV $\quad$ Aufgaben -471 
V Der harmonische Oszillator -477

Übersicht über die Ergänzungen zu Kapitel V — 503

Av Beispiele für harmonische Oszillatoren - 505

BV Stationäre Zustände. Hermitesche Polynome - 524

$C_{V} \quad$ Lösung der Eigenwertgleichung mit der Polynommethode -531

DV Stationäre Zustände in der Impulsdarstellung - 539

Ev Dreidimensionaler isotroper harmonischer Oszillator -544

Fv Geladener harmonischer Oszillator im konstanten elektrischen Feld -549

GV Quasiklassische Zustände des Oszillators — 556

$\mathrm{HV}_{\mathrm{V}} \quad$ Eigenschwingungen gekoppelter Oszillatoren $-\mathbf{5 7 3}$

Jv Lineare Oszillatorenkette. Phononen - 584

KV Kontinuierliches System. Photonen - 603

Lv Oszillator im thermodynamischen Gleichgewicht - 619

MV Aufgaben -634

VI Der Drehimpuls in der Quantenmechanik — 639

Übersicht über die Ergänzungen zu Kapitel VI — 675

$A_{v ı} \quad$ Die Kugelflächenfunktionen - 676

$\mathrm{B}_{\mathrm{VI}} \quad$ Drehimpuls und Drehungen -689

$\mathrm{C}_{\mathrm{VI}} \quad$ Drehung zweiatomiger Moleküle -712

$D_{\mathrm{VI}} \quad$ Drehimpuls eines zweidimensionalen Oszillators -727

$\mathrm{EVI}_{\mathrm{VI}} \quad$ Geladenes Teilchen im Magnetfeld. Landau-Niveaus — 742

FVI $\quad$ Aufgaben -766

VII Teilchen in einem Zentralpotential. Das Wasserstoffatom — 775

Übersicht über die Ergänzungen zu Kapitel VII — 806

AvII Wasserstoffartige Systeme -807

B $\quad$ Der dreidimensionale isotrope harmonische Oszillator -817 
CVII Wahrscheinlichkeitsströme der stationären Zustände des Wasserstoffatoms -827

D DII Das Wasserstoffatom im homogenen Magnetfeld. Paramagnetismus und Diamagnetismus. Der Zeeman-Effekt — 831

EVII Einige Atomorbitale. Hybridorbitale -844

FvII Vibrations- und Rotationsniveaus zweiatomiger Moleküle $-\mathbf{8 6 0}$

GVII $\quad$ Aufgaben -874

Bibliographie - 877

Sach- und Namenverzeichnis - 899 